\title{
Monotone and fast computation of Euler's constant
}

\author{
José A Adell ${ }^{* \dagger}$ and Alberto Lekuona ${ }^{\dagger}$
}

${ }^{\text {*Correspondence: adell@unizar.es }}$ Departamento de Métodos

Estadísticos, Facultad de Ciencias, Universidad de Zaragoza, Pedro

Cerbuna 12, Zaragoza, 50009, Spain

†Equal contributors

\section{Introduction}

The Euler-Mascheroni constant $\gamma$ was first introduced by Leonhard Euler (1707-1783) as

$$
\gamma=\lim _{n \rightarrow \infty}\left(H_{n}-\log n\right)=0.5772156 \ldots, \quad H_{n}=\sum_{k=1}^{n} \frac{1}{k}, n=1,2, \ldots
$$

This constant appears in different mathematical subjects, such as number theory, special functions, random matrix theory, random permutations, and mathematical physics, among many others. We refer the interested reader to the survey paper by Lagarias [1].

As far as we know, two main types of computations of the Euler constant have been developed. The first one emphasizes the monotonicity of the corresponding convergent sequences, but the rates of convergence are relatively slow (polynomial rates). The second one emphasizes the speed of convergence (geometric rates), but looses the monotonicity in the approximation.

In this paper, we gather both points of view by providing approximating sequences that converge at the geometric rate $1 / 2$ and satisfy complete monotonicity-type properties (monotonicity, convexity, etc.). In addition, such approximating sequences are easy to compute.

With respect to the first type of computations, we mention that $\mathrm{Xu}$ and You [2] and Lu et al. [3, 4] have used continued fractions to obtain monotone convergence to $\gamma$. For instance, it is shown in [2], Theorem 2, that

$$
\frac{C}{(n+1)^{11}}<\gamma-r_{n}<\frac{C}{n^{11}}, \quad \frac{C^{\star}}{(n+1)^{12}}<r_{n}^{\star}-\gamma<\frac{C^{\star}}{n^{12}},
$$

(c) The Author(s) 2017. This article is distributed under the terms of the Creative Commons Attribution 4.0 International License (http://creativecommons.org/licenses/by/4.0/), which permits unrestricted use, distribution, and reproduction in any medium, provided you give appropriate credit to the original author(s) and the source, provide a link to the Creative Commons license, and indicate if changes were made. 
where $C$ and $C^{\star}$ are explicit constants, and $\left(r_{n}\right)_{n \geq 1}$ and $\left(r_{n}^{\star}\right)_{n \geq 1}$ are sequences involving the logarithm of a continued fraction, the first one being strictly increasing, and the second one strictly decreasing. Yang [5] has found the constants $\left(a_{i}\right)_{0 \leq i \leq s}$ for a given $s=0,1, \ldots$ such that

$$
H_{n}-\log \left(n+\sum_{i=0}^{s} \frac{a_{i}}{n^{i}}\right)=\gamma+O\left(\frac{1}{n^{s+2}}\right) \quad \text { as } n \rightarrow \infty
$$

is the fastest sequence converging to $\gamma$, giving in this way a constructive answer to a problem posed by Chen and Mortici [6]. It turns out that, for small values of $s$, the sequence on the left-hand side in (3) strictly increases to $\gamma$.

With regard to the second type of computations, K. and T. Hessami Pilehrood [7] have provided a rational approximation $p_{n} / q_{n}$ converging to $\gamma$ subexponentially. In fact, these authors have shown that

$$
\frac{p_{n}}{q_{n}}-\gamma=e^{-4 \sqrt{n}}\left(2 \pi+O\left(n^{-1 / 2}\right)\right) \quad \text { as } n \rightarrow \infty
$$

where

$$
q_{n}=\sum_{k=0}^{n}\left(\begin{array}{l}
n \\
k
\end{array}\right)^{2} k !, \quad p_{n}=\sum_{k=0}^{n}\left(\begin{array}{l}
n \\
k
\end{array}\right)^{2} k !\left(2 H_{n-k}-H_{k}\right), \quad n=1,2, \ldots
$$

Exponential convergence to $\gamma$ is possible at the price of using logarithms. In this respect, Karatsuba [8] showed that

$$
\gamma=1-\sum_{k=1}^{12 n+1} \frac{(-1)^{k-1} n^{k+1}}{(k-1) !(k+1)}\left(\log n-\frac{1}{k+1}\right)+O\left(2^{-n}\right)
$$

whereas Coffey [9] gave the formula

$$
\gamma=\frac{\log 2}{2}-\frac{2}{3 \log 2} \sum_{k=1}^{\infty} \frac{1}{3^{k}} \sum_{j=1}^{k}(-1)^{j}\left(\begin{array}{l}
k \\
j
\end{array}\right) 2^{j} \frac{\log (j+1)}{j+1}
$$

where the series in (6) has actually the geometric rate $1 / 3$. On the other hand, Sondow [10] obtained the expression

$$
\gamma=\frac{A_{n}-L_{n}}{\left(\begin{array}{c}
2 n \\
n
\end{array}\right)}+O\left(2^{-6 n} n^{-1 / 2}\right) \quad \text { as } n \rightarrow \infty
$$

where, for any $n=1,2, \ldots$,

$$
A_{n}=\sum_{i=0}^{n}\left(\begin{array}{c}
n \\
i
\end{array}\right)^{2} H_{n+i}, \quad L_{n}=2 \sum_{0 \leq i<j \leq n} \sum_{k=1}^{j-i} \frac{(-1)^{i+j-1}}{j-i}\left(\begin{array}{c}
n \\
i
\end{array}\right)\left(\begin{array}{l}
n \\
j
\end{array}\right) \log (n+i+k) .
$$

As mentioned before, the aim of this paper is to compute Euler's constant in a monotone and fast way at the same time. To achieve this, we combine a formula obtained by Zhang and Williams [11] to compute Stieltjes constants (see also Coffey [9]) and a probabilistic 
perspective based on a differentiation formula for expectations of functions of the gamma process (see formula (20) in Section 3). More precisely, let

$$
\eta(z)=\sum_{m=0}^{\infty} \frac{(-1)^{m}}{(m+1)^{z}}, \quad \Re(z)>0,
$$

be the alternating zeta function. It was shown in Zhang and Williams [11], Theorem 4 (see also Coffey [9]) that

$$
\gamma=\frac{\log 2}{2}+\frac{\eta^{\prime}(1)}{\log 2}
$$

Our computation of $\eta^{\prime}(1)$ is mainly based on the probabilistic representation given in Lemma 3.1 (Section 3).

We point out that some authors have introduced probabilistic tools to deal with different topics of analytic number theory. For instance, Sun [12] described Stirling series in terms of products of uniformly distributed random variables, Srivastava and Vignat [13] have given representations of the Bernoulli, Euler, and Gegenbauer polynomials in terms of moments of appropriate random variables, and $\mathrm{Ta}$ [14] has recently introduced a nice probabilistic approach to study Appell polynomials by connecting them to moments of random variables. Finally, fast computations of the Stieltjes constants using differentiation formulas for linear operators represented by stochastic processes can be found in [15] and the references therein.

\section{Main results}

Denote by $\mathbb{N}$ the set of nonnegative integers, and let $\mathbb{N}_{+}=\mathbb{N} \backslash\{0\}$. The $m$ th forward differences of any sequence $\left(v_{n}\right)_{n \geq 0}$ of real numbers are recursively defined by $\Delta^{0} v_{n}=v_{n}$, $\Delta^{1} v_{n}=v_{n+1}-v_{n}, n \in \mathbb{N}$, and

$$
\Delta^{m} v_{n}=\Delta^{1}\left(\Delta^{m-1} v_{n}\right)=\sum_{j=0}^{m}\left(\begin{array}{c}
m \\
j
\end{array}\right)(-1)^{m-j} v_{n+j}, \quad m \in \mathbb{N}_{+}, n \in \mathbb{N} .
$$

Let $n \in \mathbb{N}$. We consider the coefficients

$$
a_{n}(j)=\sum_{k=j}^{n}\left(\begin{array}{c}
k+1 \\
j+1
\end{array}\right) \frac{1}{2^{k+2}}, \quad j=0,1, \ldots, n,
$$

and define the following lower and upper approximants of $\eta^{\prime}(1)$ :

$$
l_{n}=\sum_{j=0}^{n} a_{n}(j) \frac{(-1)^{j} \log (j+2)}{j+2}
$$

and

$$
u_{n}=l_{n}+\frac{n+1}{2^{n+2}} \sum_{j=0}^{n+1}\left(\begin{array}{c}
n+1 \\
j
\end{array}\right) \frac{(-1)^{j} \log (j+1)}{(j+1) j},
$$

respectively, where $\log 1 / 0:=1$. With these notations, we enunciate our first main result. 
Theorem 2.1 Let $n \in \mathbb{N}$. Then,

$$
\tilde{l}_{n}:=\frac{\log 2}{2}+\frac{l_{n}}{\log 2}<\gamma<\frac{\log 2}{2}+\frac{u_{n}}{\log 2}=: \tilde{u}_{n} .
$$

The sequences $\left(l_{n}\right)_{n \geq 0}$ and $\left(u_{n}\right)_{n \geq 0}$ satisfy the following complete monotonicity-type properties

$$
(-1)^{m-1} \Delta^{m} l_{n} \geq 0, \quad(-1)^{m-1} \Delta^{m} u_{n} \leq 0, \quad 1 \leq m \leq n+3 .
$$

In addition, we have

$$
u_{n}-l_{n} \leq \frac{n+1}{2^{n+2}} .
$$

The sequences $\left(\tilde{l}_{n}\right)_{n \geq 0}$ and $\left(\tilde{u}_{n}\right)_{n \geq 0}$ in Theorem 2.1 provide monotone and fast computations of the Euler-Mascheroni constant $\gamma$. Moreover, such sequences are easy to compute. In this regard, let $S_{j}, j \in \mathbb{N}_{+}$, be a random variable having the negative binomial distribution with parameters $j$ and $1 / 2$, that is,

$$
P\left(S_{j}=l\right)=\left(\begin{array}{c}
j-1+l \\
j-1
\end{array}\right) \frac{1}{2^{j+l}}, \quad l \in \mathbb{N} .
$$

The coefficients $a_{n}(j)$ in (10) can be represented as

$$
a_{n}(j)=\sum_{l=0}^{n-j}\left(\begin{array}{c}
j+1+l \\
j+1
\end{array}\right) \frac{1}{2^{j+2+l}}=P\left(S_{j+2} \leq n-j\right), \quad n \in \mathbb{N}, j=0, \ldots, n
$$

Thus, the tail probabilities $a_{n}(j)$ can be precomputed, as done in many statistical packages, such as R. Finally, recall that a sequence $\left(v_{n}\right)_{n \geq 0}$ is said to be completely monotonic if $(-1)^{m} \Delta^{m} v_{n} \geq 0, m, n \in \mathbb{N}$. This is the reason why the inequalities in (14) are called complete monotonicity-type properties.

The approximating sequences to $\gamma$ given in (2) and (3) are simpler to compute than those in Theorem 2.1. However, the sequences $\left(\tilde{l}_{n}\right)_{n \geq 0}$ and $\left(\tilde{u}_{n}\right)_{n \geq 0}$ in this theorem converge to $\gamma$ in a much faster way and enjoy properties such as monotonicity, convexity, and so on. On the other hand, formulas (4), (5), (6), and (7) provide fast computations of $\gamma$ at the price of loosing the monotonicity of the corresponding approximating sequences. Certainly, formula (7) computes $\gamma$ in a faster way than that in (13). However, the sequences $\left(\tilde{l}_{n}\right)_{n \geq 0}$ and $\left(\tilde{u}_{n}\right)_{n \geq 0}$ in Theorem 2.1 are easier to compute than the main term in (7).

Denote

$$
P_{k}=(\text { even }) \prod_{j=0}^{k}(j+2)^{\left(\begin{array}{c}
k+2 \\
j+2
\end{array}\right)}, \quad Q_{k}=(\text { odd }) \prod_{j=0}^{k}(j+2)^{\left(\begin{array}{c}
k+2 \\
j+2
\end{array}\right)}, \quad k \in \mathbb{N},
$$

where (even) $\prod$ (resp. (odd) $\prod$ ) means that the product is extended to those even (resp. odd) integers $j$ running from 0 to $k$. As a consequence of Theorem 2.1, we give the following: 
Corollary 2.2 We have the infinite product representation

$$
2^{\gamma-\log 2 / 2}=\lim _{n \rightarrow \infty} \prod_{k=0}^{n}\left(\frac{P_{k}}{Q_{k}}\right)^{1 /(k+2) 2^{k+2}},
$$

where $P_{k} / Q_{k}>1, k \in \mathbb{N}$.

Guillera and Sondow [16], Example 5.8, have obtained the product formula

$$
e^{\gamma-\log 2 / 2}=\lim _{n \rightarrow \infty} \prod_{k=0}^{n}\left(\frac{Q_{k}^{\star}}{P_{k}^{\star}}\right)^{1 /(k+3)}
$$

where

$$
P_{k}^{\star}=(\text { even }) \prod_{j=0}^{k+2}(j+1) \stackrel{\left(\begin{array}{c}
k+2 \\
j
\end{array}\right), \quad Q_{k}^{\star}=(\text { odd })}{k+2} \prod_{j=0}^{k+2}(j+1)^{\left(\begin{array}{c}
k+2 \\
j
\end{array}\right)}, \quad k \in \mathbb{Z}_{+} .
$$

However, the rate of convergence in Corollary 2.2 is faster than that in (17).

\section{Auxiliary results}

Let $\left(X_{t}\right)_{t \geq 0}$ be a gamma process (see Çınlar [17], pp.279-281), that is, a stochastic process starting at the origin, having independent stationary increments, and such that for each $t>0$, the random variable $X_{t}$ has the gamma density

$$
\rho_{t}(\theta)=\frac{1}{\Gamma(t)} \theta^{t-1} e^{-\theta}, \quad \theta>0
$$

On the other hand, let $V$ and $T$ be two independent random variables such that $V$ is uniformly distributed on $[0,1]$ and $T$ has the exponential density $\rho_{1}(\theta)$ defined in (19). We assume that $V$ and $T$ are independent of the gamma process $\left(X_{t}\right)_{t \geq 0}$. Finally, let $f$ : $\mathbb{R}_{+} \rightarrow \mathbb{R}$ be a differentiable function such that $f_{\star}(t):=E f\left(X_{t}\right)<\infty, t \geq 0$, where $E$ stands for mathematical expectation. It has been shown in [18], Theorem 7.1, that

$$
f_{\star}^{\prime}(t)=E f^{\prime}\left(X_{t}+V T\right), \quad t \geq 0 .
$$

This formula can be applied to the problem at hand as follows.

Lemma 3.1 Let $\eta$ be the alternating zeta function. Then,

$$
\eta^{\prime}(1)=E g\left(X_{1}+V T\right),
$$

where the function $g$ is defined in $\mathbb{R}_{+}$as

$$
g(x)=\sum_{k=0}^{\infty} \frac{k+1}{2^{k+2}}\left(1-e^{-x}\right)^{k} e^{-x}=\frac{1}{4}-\sum_{k=0}^{\infty} \frac{k}{2^{k+3}}\left(1-e^{-x}\right)^{k+1} .
$$


Proof By (19) the Laplace transform of $X_{t}$ is given by

$$
E e^{-\lambda X_{t}}=\frac{1}{(\lambda+1)^{t}}, \quad \lambda \geq 0, t \geq 0 .
$$

Thus, interchanging the sum with expectation, from (8) we have

$$
\eta(t)=\sum_{m=0}^{\infty} E\left(-e^{-X_{t}}\right)^{m}=E f\left(X_{t}\right), \quad t \geq 0
$$

where

$$
f(x)=\frac{1}{1+e^{-x}}=\sum_{k=0}^{\infty} \frac{\left(1-e^{-x}\right)^{k}}{2^{k+1}}, \quad x \geq 0 .
$$

Therefore from (20) and (24) we have

$$
\eta^{\prime}(1)=E f^{\prime}\left(X_{1}+V T\right)
$$

This shows (21), since $f^{\prime}=g$, as follows from (25). Finally, the second equality in (22) follows by calculus. The proof is complete.

In view of Lemma 3.1, we define, for any $n \in \mathbb{N}$ and $x \geq 0$,

$$
L_{n}(x)=\sum_{k=0}^{n} \frac{k+1}{2^{k+2}}\left(1-e^{-x}\right)^{k} e^{-x}, \quad U_{n}(x)=\frac{1}{4}-\sum_{k=0}^{n-1} \frac{k}{2^{k+3}}\left(1-e^{-x}\right)^{k+1} .
$$

It can be checked from (26) that

$$
L_{n}(x)=U_{n}(x)-\frac{n+1}{2^{n+2}}\left(1-e^{-x}\right)^{n+1} .
$$

Such partial sums allow us to give the following probabilistic representations of the sequences $\left(l_{n}\right)_{n \geq 0}$ and $\left(u_{n}\right)_{n \geq 0}$ respectively defined in (11) and (12).

Lemma 3.2 For any $n \in \mathbb{N}$, we have

$$
l_{n}=E L_{n}\left(X_{1}+V T\right), \quad u_{n}=E U_{n}\left(X_{1}+V T\right) .
$$

Proof By Fubini's theorem the Laplace transform of $V T$ is given by

$$
E e^{-\lambda V T}=E \frac{1}{\lambda V+1}=\frac{\log (\lambda+1)}{\lambda}, \quad \lambda \geq 0 .
$$

Since $X_{1}$ is independent of $V T$, from (23) and (28) we have

$$
\begin{aligned}
E L_{n}\left(X_{1}+V T\right) & =\sum_{k=0}^{n} \frac{k+1}{2^{k+2}} \sum_{j=0}^{k}\left(\begin{array}{l}
k \\
j
\end{array}\right)(-1)^{j} E e^{-(j+1)\left(X_{1}+V T\right)} \\
& =\sum_{k=0}^{n} \frac{k+1}{2^{k+2}} \sum_{j=0}^{k}\left(\begin{array}{l}
k \\
j
\end{array}\right) \frac{(-1)^{j} \log (j+2)}{(j+2)(j+1)}=l_{n},
\end{aligned}
$$


where the last equality follows from (10) and (11) after interchanging the order of summation. Similarly,

$$
E\left(1-e^{-\left(X_{1}+V T\right)}\right)^{n+1}=\sum_{j=0}^{n+1}\left(\begin{array}{c}
n+1 \\
j
\end{array}\right) \frac{(-1)^{j} \log (j+1)}{(j+1) j} .
$$

Therefore, the second equality in Lemma 3.2 follows from (12), (27), and (29). The proof is complete.

Thanks to Lemma 3.2, the complete monotonicity-type properties of $\left(l_{n}\right)_{n \geq 0}$ and $\left(u_{n}\right)_{n \geq 0}$ are easily derived from the analogous properties satisfied by the sequences of functions $\left(L_{n}(x)\right)_{n \geq 0}$ and $\left(U_{n}(x)\right)_{n \geq 0}$.

Lemma 3.3 Let $m \in \mathbb{N}_{+}$and $n \in \mathbb{N}$ with $m \leq n+3$. Then,

$$
(-1)^{m-1} \Delta^{m} L_{n}(x) \geq 0, \quad(-1)^{m-1} \Delta^{m} U_{n}(x) \leq 0, \quad x \geq 0 .
$$

Proof Fix $x \geq 0$. For any $s \geq 0$, denote

$$
v_{n}(s)=(n+s) \tau^{n}(x), \quad \tau(x)=\frac{1-e^{-x}}{2}, n \in \mathbb{N} .
$$

Using induction on $m$, we can check that

$$
(-1)^{m} \Delta^{m} v_{n}(s)=\tau^{n}(x)(1-\tau(x))^{m-1}(n+s-(n+s+m) \tau(x)), \quad n, m \in \mathbb{N},
$$

thus implying that

$$
(-1)^{m} \Delta^{m} v_{n}(s) \geq 0, \quad m, n \in \mathbb{N}, m \leq n+s
$$

since $0 \leq \tau(x) \leq 1 / 2$. On the other hand, from (26) and (30) we have

$$
\Delta^{1} L_{n}(x)=\frac{e^{-x}}{4}(n+2) \tau^{n+1}(x)=\frac{e^{-x}}{4} v_{n+1}(1) .
$$

Therefore, the first inequality in Lemma 3.3 follows from (31). Finally, from (26) and (30) we have

$$
\Delta^{1} U_{n}(x)=-\frac{n}{4} \tau^{n+1}(x)=-\frac{\tau(x)}{4} \nu_{n}(0) .
$$

This, together with (31), shows the second inequality in Lemma 3.3 and completes the proof.

\section{The proofs}

Proof of Theorem 2.1 Let $n \in \mathbb{N}$. By (26) and Lemmas 3.1 and 3.2 we get

$$
l_{n}=E L_{n}\left(X_{1}+V T\right)<E g\left(X_{1}+V T\right)=\eta^{\prime}(1)<E U_{n}\left(X_{1}+V T\right)=u_{n} .
$$


This, in conjunction with (9), shows (13). On the other hand, let $m \in \mathbb{N}_{+}$with $1 \leq m \leq n+3$. By Lemmas 3.2 and 3.3 we have

$$
(-1)^{m-1} \Delta^{m} l_{n}=E(-1)^{m-1} \Delta^{m} L_{n}\left(X_{1}+V T\right) \geq 0 .
$$

The second inequality in (14) is shown in a similar way. Finally, we see from (27) and Lemma 3.2 that

$$
u_{n}-l_{n}=\frac{n+1}{2^{n+2}} E\left(1-e^{-\left(X_{1}+V T\right)}\right)^{n+1} \leq \frac{n+1}{2^{n+2}} .
$$

The proof is complete.

Proof of Corollary 2.2 Let $k \in \mathbb{N}$. Using (16), (23), and (28), we can check that

$$
\begin{aligned}
\log \frac{P_{k}}{Q_{k}} & =\sum_{j=0}^{k}\left(\begin{array}{c}
k+2 \\
j+2
\end{array}\right)(-1)^{j} \log (j+2) \\
& =(k+2)(k+1) E\left\{\left(1-e^{-\left(X_{1}+V T\right)}\right)^{k} e^{-\left(X_{1}+V T\right)}\right\}>0,
\end{aligned}
$$

which implies that $P_{k} / Q_{k}>1$. Therefore, for any $n \in \mathbb{N}$, we have

$$
\log \prod_{k=0}^{n}\left(\frac{P_{k}}{Q_{k}}\right)^{1 /(k+2) 2^{k+2}}=\sum_{k=0}^{n} \frac{1}{2^{k+2}} \sum_{j=0}^{k}\left(\begin{array}{c}
k+1 \\
j+1
\end{array}\right) \frac{(-1)^{j} \log (j+2)}{j+2}=l_{n},
$$

where the last equality follows from (10) and (11). This, together with (13) and (15), shows the result.

\section{Acknowledgements}

The authors are partially supported by Research Projects DGA (E-64), MTM2015-67006-P, and by FEDER funds.

\section{Competing interests}

The authors declare that they have no competing interests.

\section{Authors' contributions}

Both authors read and approved the final manuscript.

\section{Publisher's Note}

Springer Nature remains neutral with regard to jurisdictional claims in published maps and institutional affiliations.

Received: 3 May 2017 Accepted: 30 August 2017 Published online: 15 September 2017

\section{References}

1. Lagarias, JC: Euler's constant: Euler's work and modern developments. Bull. Am. Math. Soc. (N.S.) 50(4), 527-628 (2013). doi:10.1090/S0273-0979-2013-01423-X

2. $\mathrm{Xu}, \mathrm{H}, \mathrm{You}, \mathrm{X}$ : Continued fraction inequalities for the Euler-Mascheroni constant. J. Inequal. Appl. 2014, Article ID 343 (2014). doi:10.1186/1029-242X-2014-343

3. Lu, D, Song, L, Yu, Y: Some new continued fraction approximation of Euler's constant. J. Number Theory 147, 69-80 (2015). doi:10.1016/j.jnt.2014.07.002

4. Lu, D, Song, L, Yu, Y: Some quicker continued fraction approximations and inequalities towards Euler's constant. J. Number Theory 175, 100-116 (2017). doi:10.1016/j.jnt.2016.11.024

5. Yang, S: On an open problem of Chen and Mortici concerning the Euler-Mascheroni constant. J. Math. Anal. Appl. 396(2), 689-693 (2012). doi:10.1016/j.jmaa.2012.07.007

6. Chen, C-P, Mortici, C: New sequence converging towards the Euler-Mascheroni constant. Comput. Math. Appl. 64(4), 391-398 (2012). doi:10.1016/j.camwa.2011.03.099

7. Hessami Pilehrood, Kh, Hessami Pilehrood, T: On a continued fraction expansion for Euler's constant. J. Number Theory 133(2), 769-786 (2013). doi:10.1016/j.jnt.2012.08.016 
8. Karatsuba, EA: On the computation of the Euler constant $\gamma$. Numer. Algorithms 24(1-2), 83-97 (2000). doi:10.1023/A:1019137125281

9. Coffey, MW: The Stieltjes constants, their relation to the $\eta_{j}$ coefficients, and representation of the Hurwitz zeta function. Analysis 30(4), 383-409 (2010). doi:10.1524/anly.2010.1048

10. Sondow, J: Criteria for irrationality of Euler's constant. Proc. Am. Math. Soc. 131(11), 3335-3344 (2003). doi:10.1090/S0002-9939-03-07081-3

11. Zhang, NY, Williams, KS: Some results on the generalized Stieltjes constants. Analysis 14(2-3), 147-162 (1994)

12. Sun, P: Product of uniform distribution and Stirling numbers of the first kind. Acta Math. Sin. Engl. Ser. 21(6), 1435-1442 (2005). doi:10.1007/s10114-005-0631-4

13. Srivastava, HM, Vignat, C: Probabilistic proofs of some relationships between the Bernoulli and Euler polynomials. Eur. J. Pure Appl. Math. 5(2), 97-107 (2012)

14. Ta, BQ: Probabilistic approach to Appell polynomials. Expo. Math. 33(3), 269-294 (2015). doi:10.1016/j.exmath.2014.07.003

15. Adell, JA: Estimates of generalized Stieltjes constants with a quasi-geometric rate of decay. Proc. R. Soc., Math. Phys. Eng. Sci. 468(2141), 1356-1370 (2012). doi:10.1098/rspa.2011.055

16. Guillera, J, Sondow, J: Double integrals and infinite products for some classical constants via analytic continuations of Lerch's transcendent. Ramanujan J. 16(3), 247-270 (2008). doi:10.1007/s11139-007-9102-0

17. Çınlar, E: Probability and Stochastics. Graduate Texts in Mathematics, vol. 261, p. xiv+557. Springer, Berlin (2011). doi:10.1007/978-0-387-87859-1.

18. Adell, JA: Differential calculus for linear operators represented by finite signed measures and applications. Acta Math. Hung. 138(1-2), 44-82 (2013). doi:10.1007/s10474-012-0230-7

\section{Submit your manuscript to a SpringerOpen ${ }^{\circ}$ journal and benefit from:}

- Convenient online submission

- Rigorous peer review

Open access: articles freely available online

High visibility within the field

- Retaining the copyright to your article

Submit your next manuscript at $\gg$ springeropen.com 